\title{
Suprainguinal ectopic scrota of TS inbred rats
}

\author{
H. Ikadai, C. Ajisawa, K. Taya* and T. Imamichi \\ The Imamichi Institute for Animal Reproduction, 1103 Dejima-mura Fukaya, Niihari-gun, Ibaraki \\ 300-01, Japan; and ${ }^{*}$ Laboratory of Veterinary Physiology, Tokyo University of Agriculture and \\ Technology, Fuchu, Tokyo 183, Japan
}

\begin{abstract}
Summary. A new rat line (TS inbred rats) showing uni- or bilateral ectopic scrota in about $70 \%$ of the males was established. Ectopic scrota were elongated pouches of the thin muscle layer under the suprainguinal body skin, in which hypoplastic testes and epididymides were seen. Genetic analysis revealed that the ectopic scrotum was controlled by multiple genes. Groups of 5 normal, uni- and bilaterally affected rats were killed at 25 weeks of age. Histologically, ectopic testes of uni- and bilaterally affected rats showed arrest of spermatogenesis at the primary spermatocyte stage. Contralateral testes of unilaterally affected rats were normally descended and showed normal spermatogenesis. No abnormality was seen in the urinary system. Plasma concentrations of FSH were significantly elevated in bilaterally affected rats but plasma concentrations of testosterone and $\mathrm{LH}$ were unchanged in uni- and bilaterally affected rats. Pituitary contents of LH and FSH were significantly elevated in bilaterally affected rats. The endocrinological status of TS inbred rats was therefore similar to that of experimentally induced cryptorchid rats.
\end{abstract}

Keywords: ectopic scrotum; inheritance; rat; testis; LH; FSH; testosterone

\section{Introduction}

There are very few reports of ectopic scrota and the pathogenesis of the condition has not been fully understood (Lamm \& Kaplan, 1977; Elder \& Jeffs, 1982). Ectopic scrota in rodents have not been reported in the literature. The occurrence of uni- or bilateral ectopic scrota in a colony of rats has been found and a line manifesting the defect in about $70 \%$ of the males has been established. The suprainguinal body wall makes a pouch instead of the normal scrotum and testes and epididymides are seen in the pouch. In this paper we describe the morphological and hormonal features and the mode of inheritance.

\section{Materials and Methods}

Animals. Rats showing uni- or bilateral ectopic scrota were detected in 1984 in a colony of the WKA rat strain, which had been maintained in the Imamichi Institute. Since bilaterally affected rats were infertile, unilaterally affected rats were mated with sibling females and successive inbreedings have been made for 7 generations. The incidence of affected rats was gradually raised in the line designated as TS and reached $73 \%$ of males in the 7 th generation, in which 11 rats were affected on the right side, 13 rats on the left side, and 5 rats on both sides out of 40 males. Animals were kept under conventional conditions and were fed a commercial diet (EM; Oriental-kobo, Co. Ltd, Tokyo) and tap water ad libitum.

Blood and pituitary collection and testicular histology. After weighing, groups of 5 normal, uni- or bilaterally affected TS rats were killed by decapitation at 25 weeks of age. Blood was collected and serum saved at $-20^{\circ} \mathrm{C}$ for radioimmunoassay (RIA) of testosterone, luteinizing hormone (LH) and follicle-stimulating hormone (FSH). Anterior pituitary glands removed immediately after death were homogenized in $4 \mathrm{ml}$ cold saline $(8 \cdot 5 \mathrm{~g} \mathrm{NaCl} / \mathrm{l})$. After centrifugation at $36000 \mathrm{~g}$ for $30 \mathrm{~min}$ at $4^{\circ} \mathrm{C}$, the supernatant fraction was saved at $-20^{\circ} \mathrm{C}$ for RIA of LH and FSH. The testes, epididymides, seminal vesicles, ventral prostate, preputial glands, kidneys, thymus, and pituitary gland 
were removed and weighed immediately. Testes were fixed in Bouin's solution, embedded in paraffin wax, sectioned, and stained with haematoxylin and eosin for microscopic observations.

Measurement of scrotal and abdominal temperature. Five adult unilaterally affected rats were anaesthetized with pentobarbitone sodium and 3 small incisions were made in the skin superficial to the bilateral external inguinal ring and mid-abdomen in each rat. Probes of digital electric thermisters (PC-700, Satoh keiryouki Co. Ltd, Tokyo) were passed through each incision and were placed in the abdominal cavity, the normal scrotum and the ectopic scrotum. After stabilization ( $\sim 5 \mathrm{~min}$ ), each temperature was recorded simultaneously.

Hormone assays. Serum and pituitary concentrations of FSH and LH were measured using NIADDK RIA kits for rat FSH and rat LH. Iodinated preparations were rat FSH-I-5 and LH-I-5. The antisera used were anti-rat FSH-S-11 and anti-rat LH-S-8. Results were expressed in terms of the NIADDK rat FSH-RP-1 and LH-RP-1 preparations. The sensitivity in the assay for LH and FSH were 0.25 and $1.2 \mathrm{ng} /$ tube and the intra- and inter-assay coefficients of variation were 4.8 and $6.8 \%$ for FSH and 6.5 and $11.1 \%$ for $\mathrm{LH}$ respectively.

Serum concentrations of testosterone were measured by a double-antibody RIA system using a ${ }^{125}$ I-labelled radioligand as described previously (Taya et al., 1985). The antiserum against testosterone (GDN 250; Gay \& Kerlen, 1978) was provided by Dr G. D. Niswender (Colorado State University, Fort Collins, CO, U.S.A.); the sensitivity of the assay was $0.625 \mathrm{pg} /$ tube and the intra- and inter-assay coefficients of variation were 6.0 and 15.5 respectively.

Genetic analyses. To elucidate the mode of inheritance of ectopic scrota, unilaterally affected males and dams proven to produce the affected offspring were reciprocally mated with rats in an unrelated line, the NIG III line, that was provided by Dr T. Yoshida (The National Institute of Genetics, Shizuoka, Japan) in 1983 and has been inbred in the Institute. Segregation of the affected rats in the F1, F2, back-cross, and back-back-cross generations was investigated.

Statistical analysis. Values were expressed as mean \pm s.e.m. Statistical comparisons were made with Student's $t$ test. A $P$ value of $<0.05$ was selected as the limit of statistical significance.

\section{Results}

\section{Morphological features (Fig. 1).}

Ectopic scrota were detectable in appearance after 3 weeks of age by swellings of the suprainguinal body wall. They appeared as elongated pouches of the muscle layer continuous with the abdominal wall at the inguinal region and separable from the body wall. The pouch extended about $2 \mathrm{~cm}$ from the inguinal region in a cranial direction, and opened into the peritoneal cavity through the inguinal canal. The ipsilateral scrotum was not formed. Testes and epididymides were seen in the pouch and were easily pulled out into the body cavity. Contralateral testes in unilaterally affected rats descended as usual into the normally formed scrotum. No abnormality was seen in the kidneys, ureters, bladder and penis. Female litter mates showed no defect.

\section{Body and organ weights (Table 1)}

In the present experiment, body weight was significantly lower in unilaterally affected rats than in normal rats. Weights of testes and epididymides on the ectopic side of uni- and bilaterally affected rats were about one-half those of normal rats. The same tissues from the normally descended side in unilaterally affected rats were not significantly different compared with those of normal rats. Weights of the ventral prostate, preputial glands and seminal vesicles of bilaterally affected rats were significantly lower and weight of thymus was significantly higher than those of normal rats. Weights of the ventral prostate and seminal vesicles of unilaterally affected rats were also significantly smaller than those of normal rats.

\section{Testicular histology (Fig. 2)}

Spermatogenesis was arrested at the primary spermatocyte stage in bilaterally affected rats. The diameter of seminiferous tubules was decreased and Sertoli cells with shrunken nuclei had separated from basement of the tubule. Oedema was prominent in the interstitial tissue and the numbers of Leydig cells appeared to be increased. Testes on the ectopic side of unilaterally affected rats showed histology similar to that of bilaterally affected rats, whereas the descended testes of these rats showed spermatogenesis as seen in normal rats. 


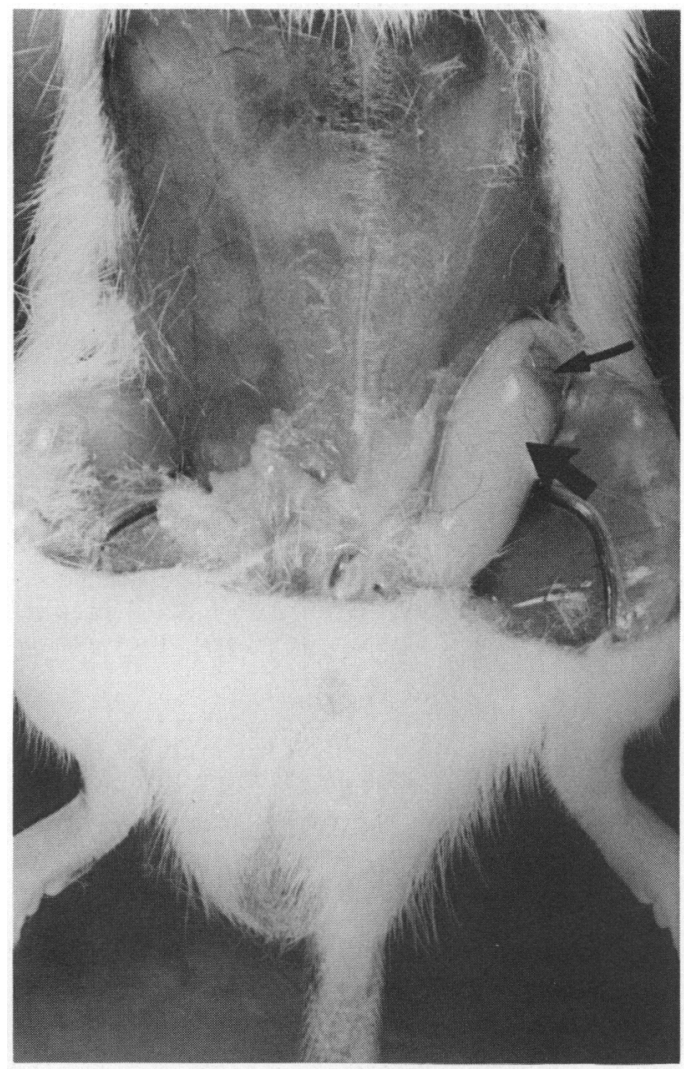

Fig. 1. A rat with a unilateral ectopic scrotum. The left scrotum (large arrow) is affected and the right scrotum is normal. A small arrow indicates the caudal end of the ectopic scrotum and testis.

Table 1. Body and organ weights of normal and TS inbred rats showing uni- or bilateral ectopic scrota

\begin{tabular}{|c|c|c|c|}
\hline \multirow[b]{3}{*}{ Body weight (g) } & \multirow[b]{2}{*}{ Normal rats } & \multicolumn{2}{|c|}{ TS rats } \\
\hline & & $\begin{array}{l}\text { Unilaterally } \\
\text { affected }\end{array}$ & $\begin{array}{l}\text { Bilaterally } \\
\text { affected }\end{array}$ \\
\hline & $478 \cdot 0 \pm 18 \cdot 2$ & $425 \cdot 6 \pm 12 \cdot 7^{*}$ & $446.0 \pm 8.5$ \\
\hline Pituitary gland (mg) & $14.0 \pm 0.7$ & $12.7 \pm 0.6$ & $13.4 \pm 0.2$ \\
\hline Thymus (mg) & $129.8 \pm 14.6$ & $175.8 \pm \quad 26.9$ & $261 \cdot 2 \pm 26 \cdot 0^{*}$ \\
\hline Kidneys (mg) & $2727.8 \pm 127.9$ & $2623 \cdot 8 \pm 125 \cdot 5$ & $2668 \cdot 6 \pm 79 \cdot 6$ \\
\hline Ventral prostate $(\mathrm{mg})$ & $551 \cdot 8 \pm 44 \cdot 6$ & $422.0 \pm 31 \cdot 4^{*}$ & $339 \cdot 6 \pm 37 \cdot 0^{*}$ \\
\hline Preputial glands (mg) & $281 \cdot 0 \pm 24.0$ & $233.8 \pm 34.6$ & $155.0 \pm 6.4^{*}$ \\
\hline Seminal vesicles (mg) & $1396.0 \pm 77.7$ & $976.8 \pm 73.1^{*}$ & $866^{2} \pm 79 \cdot 8^{*}$ \\
\hline Epididymis (mg) & $703.9 \pm 12.7(10)$ & $\begin{array}{c}386.8 \pm 16.1^{*} \\
(695.2 \pm 19.7) \dagger\end{array}$ & $445 \cdot 5 \pm 11 \cdot 5^{*}(10)$ \\
\hline Testis (mg) & $1393.4 \pm 59.4(10)$ & $\begin{aligned} 645.4 \pm & 59.3^{*} \\
(1577.0 \pm & 42.5) \dagger\end{aligned}$ & $784.7 \pm 31.7^{*}(10)$ \\
\hline
\end{tabular}

Values are mean \pm s.e.m. for 5 observations except as indicated in parentheses.

$\uparrow$ Weights of the epididymis and testis on the contralateral side of unilaterally affected rats are given in parentheses.

*Significantly different from value for normal animals $(P<0.05$, Student's $t$ test $)$. 

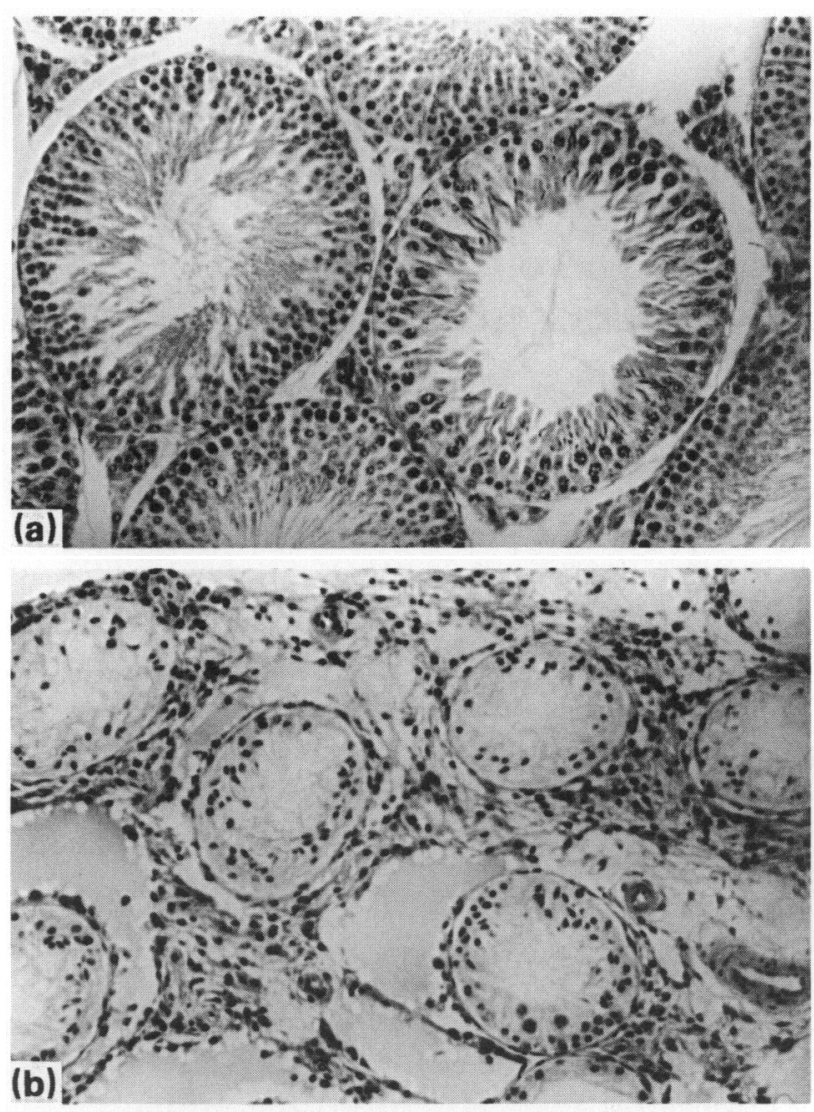

Fig. 2. Testicular histology on the normal (a) and ectopic (b) side of a unilaterally affected rat. $\times 115$.

Table 2. Serum concentrations of testosterone, LH and FSH and pituitary contents of LH and FSH in normal and TS inbred rats showing uni- or bilateral ectopic scrota

\begin{tabular}{lrrr}
\hline & & \multicolumn{2}{c}{ TS rats } \\
\cline { 3 - 4 } & Normal rats & \multicolumn{1}{c}{$\begin{array}{c}\text { Unilaterally } \\
\text { affected }\end{array}$} & \multicolumn{1}{c}{$\begin{array}{c}\text { Bilaterally } \\
\text { affected }\end{array}$} \\
\hline Serum concentration & & & \\
$\quad$ Testosterone $(\mathrm{ng} / \mathrm{ml})$ & $1 \cdot 8 \pm 0 \cdot 2$ & $1 \cdot 5 \pm 0 \cdot 3$ & $1 \cdot 4 \pm 0 \cdot 2$ \\
LH $(\mathrm{ng} / \mathrm{ml})$ & $39 \cdot 5 \pm 10 \cdot 3$ & $39 \cdot 1 \pm 6 \cdot 5$ & $57 \cdot 2 \pm 8 \cdot 8$ \\
$\quad$ FSH $(\mathrm{ng} / \mathrm{ml})$ & $279 \cdot 9 \pm 42 \cdot 7$ & $317 \cdot 4 \pm 35 \cdot 5$ & $387 \cdot 8 \pm 16 \cdot 1^{*}$ \\
Pituitary content & & & \\
$\quad$ LH $(\mu \mathrm{g} /$ pituitary) & $107 \cdot 0 \pm 7 \cdot 1$ & $136 \cdot 3 \pm 14 \cdot 0$ & $172 \cdot 5 \pm 8 \cdot 1^{*}$ \\
FSH $(\mu \mathrm{g} /$ pituitary) & $583 \cdot 2 \pm 49 \cdot 1$ & $546 \cdot 6 \pm 28 \cdot 0$ & $828.9 \pm 70 \cdot 8^{*}$ \\
\hline
\end{tabular}

Values are mean \pm s.e.m. for 5 observations.

*Significantly different from value for normal animals $(P<0.05$, Student's $t$ test). 
Temperature of ectopic scrota

The average temperatures of the abdominal cavity, normal and ectopic scrotum in unilaterally affected rats were $37 \cdot 7,31 \cdot 8$ and $36 \cdot 8^{\circ} \mathrm{C}$ respectively.

Serum concentrations of testosterone, $L H$ and FSH and pituitary content of LH and FSH (Table 2)

There was no significant difference in serum concentrations of testosterone among normal, uni- and bilaterally affected rats. Neither FSH nor LH concentration in serum and pituitary was significantly changed in unilaterally affected rats. In bilaterally affected rats, however, serum concentrations of FSH and pituitary contents of FSH and LH were significantly higher than those of normal rats. Serum concentrations of LH in bilaterally affected rats showed a tendency to be higher than those of the normal animals although the difference was not significant.

\section{Genetic analyses (Table 3)}

In the $\mathrm{F} 1$ offspring from crosses of unilaterally affected males and proven carrier dams with the NIG III line, no affected rats appeared. In the F2 generation, only 1 affected rat was observed out of 194 males and 3 out of 172 were observed in the back-cross generation. Since incidences of the disorder in the F2 and back-cross generations were very low, then the back-back-cross generation was additionally made. The incidence of ectopic scrota in the back-back-cross generation was 17 out of $189(9 \cdot 5 \%)$.

Table 3. Segregation of the ectopic scrotum (ES) in offspring from matings of TS rats with NIG III strain

\begin{tabular}{lcr}
\hline $\begin{array}{l}\text { Cross } \\
\text { (female } \times \text { male) }\end{array}$ & ES/male & $(\%)$ \\
\hline (1) NIG III $\times$ TS & $0 / 46$ & $(0)$ \\
(2) NIG III.TS $\times$ NIG III·TS & $1 / 83$ & $(1 \cdot 2)$ \\
(3) NIG III.TS $\times$ TS & $2 / 97$ & $(2 \cdot 1)$ \\
$(4)$ NIG III.TS TS $\times$ TS & $11 / 109$ & $(10 \cdot 1)$ \\
(5) TS $\times$ NIG III & $0 / 34$ & $(0)$ \\
(6) TS.NIG III $\times$ TS·NIG III & $0 / 111$ & $(0)$ \\
(7) TS $\times$ TS·NIG III & $1 / 75$ & $(1 \cdot 3)$ \\
$(8)$ TS $\times$ TS·TS·NIG III & $6 / 80$ & $(7 \cdot 5)$ \\
\hline
\end{tabular}

\section{Discussion}

The present results show that TS inbred rats are characterized by uni- or bilateral suprainguinal ectopic scrota and hypoplastic testes and epididymides on the affected side. The testes and epididymides could be easily pulled out from the ectopic scrota into the body cavity. Abnormalities were restricted to the testes and epididymides associated with the ectopic scrotum. The urinary system and penis were normal.

In the development of the scrotum and the initial step of testicular descent, the gubernaculum testis plays a major role. In rats at 16 days post coitum, the gubernaculum is seen as a mesenchymal strand (proximal part) and mass (distal part, subdivided into intra- and extra-abdominal part) connecting the testes and inguinal area. First, outgrowth in the extra-abdominal part of the gubernaculum occurs with increasing age and the proximal and intra-abdominal parts become shorter, bringing the testis and epididymis closer to the deep inguinal ring. Then, regression of the 
distal part occurs and creates the space of the future scrotum by Day 4 after birth (Wensing, 1986). An intact attachment of the distal gubernaculum to the scrotum is necessary for scrotal development and subsequent testicular migration. If the testis is excised at birth, the remaining distal part of the gubernaculum and the ipsilateral hemiscrotum can develop, but when the distal part of the gubernaculum is excised the ipsilateral hemiscrotum does not fully develop (Frey \& Rajfer, 1984). It is suggested that an ectopic scrotum results when the distal portion of the gubernaculum is misdirected (Lamm \& Kaplan, 1977; Shafik, 1977; Backhouse, 1982; Elder \& Jeffs, 1982).

Genetic analyses indicated that the ectopic scrotum in the line is a genetic disorder controlled by multiple genes. The frequencies of the abnormality in the F1, F2, and back-cross generations were very low and were not compatible with simple Mendelian transmission. The frequency in the backback-cross generation was higher than that in the back-cross generation, and the frequency in the back-cross generation was higher than that in the F2 generation. These results suggested that multiple genes are involved in this abnormality.

Arrest of spermatogenesis in the testes in the ectopic scrota may be a result of increased temperature in this scrotum. Surgically induced cryptorchidism results in a progressive degeneration of germ cells and Sertoli cells of the testis. Uni- and bilateral cryptorchidism also results in some degree of Leydig cell failure (Clegg, 1963; Kerr et al., 1979a, b). Serum concentrations of LH and FSH are elevated and serum values of testosterone were significantly lower or unchanged, and a diminished testosterone response to $\mathrm{hCG}$ stimulation is observed in vivo compared with control animals (Gomes \& Jain, 1976; de Kretser et al., 1979; Keel \& Abney, 1980; Risbridger et al., 1981a, b; Jegou et al., 1984). Previous studies have also demonstrated that there is a decrease in testicular inhibin content in uni- and bilateral cryptorchid testes (Au et al., 1983; Seethalakshmi \& Steinberger, 1983; Demura et al., 1987). The testicular histology and endocrine status of TS inbred rats are similar to those in rats with artificially induced cryptorchidism.

We thank Dr A. F. Parlow and the Rat Pituitary Hormone Distribution Program, NIADDK, NIH, MD, U.S.A., for RIA materials; Dr G. D. Niswender, Department of Physiology and Biophysics, Colorado State University, Colorado, U.S.A., for antiserum to testosterone (GDN 250); and Teikoku Hormone MFG. Co., Ltd, Tokyo, Japan, for testosterone.

\section{References}

Au, C.L., Robertson, D.M. \& de Kretser, D.M. (1983) In vitro bioassay of inhibin in testes of normal and cryptorchid rats. Endocrinology 112, 239-244.

Backhouse, K.M. (1982) Embryology of testicular descent and maldescent. Urol. Clin. North America 9, 315-325.

Clegg, E.J. (1963) Studies on artificial cryptorchidism: degenerative and regenerative changes in the germinal epithelium of the rat testis. $J$. Endocr. 27, 241-251.

de Kretser, D.M., Sharpe, R.M. \& Swanston, I.A. (1979) Alterations in steroidogenesis and human chorionic gonadotropin binding in the cryptorchid rat testis. Endocrinology 105, 135-138.

Demura, R., Suzuki, T., Nakamura, S., Komatsu, H., Jibiki, K., Odagiri, E., Demura, H. \& Shizume, $\mathbf{K}$. (1987) Effect of uni- and bilateral cryptorchidism on testicular inhibin and testosterone secretion in rats. Endocrinol. Japon. 34, 911-917.

Elder, J.S. \& Jefís, R.D. (1982) Suprainguinal ectopic scrotum and associated anomalies. J. Urol. 127, $336-338$.

Frey, H.L. \& Rajfer, J. (1984) Role of the gubernaculum and intra-abdominal pressure in the process of testicular descent. $J$. Urol. 131, 574-579.
Gay, V.L. \& Kerlen, J.T. (1978) Serum LH and FSH following passive immunization against circulating testosterone in the intact male rat and in orchidectomized rats bearing subcutaneous Silastic implants of testosterone. Archs Androl. 1, 257-266.

Gomes, W.R. \& Jain, S.K. (1976) Effect of unilateral and bilateral castration and cryptorchidism on serum gonadotrophins in the rat. J. Endocr. 68, 191-196.

Jegou, B., Peake, R.A., Irby, D.C. \& de Kretser, D.M. (1984) Effects of induction of experimental cryptorchidism and subsequent orchidopexy on testicular function in immature rats. Biol. Reprod. 30, 179-187.

Keel, B.A. \& Abney, T.O. (1980) Influence of bilateral cryptorchidism in the mature rat: alterations in testicular function and serum hormone levels. Endocrinology 107, 1226-1233.

Kerr, J.B., Rich, K.A. \& de Kretser, D.M. (1979a) Alterations of the fine structure and androgen secretion of the interstitial cells in the experimentally cryptorchid rat testis. Biol. Reprod. 20, 409422.

Kerr, J.B., Rich, K.A. \& de Kretser, D.M. (1979b) Effects of experimental cryptorchidism on the ultrastructure and function of the Sertoli cell and peritubular tissue of the rat testis. Biol. Reprod. 21, 823-838. 
Lamm, D.L. \& Kaplan, G.W. (1977) Accessory and ectopic scrota. Urology 9, 149-153.

Risbridger, G.P., Kerr, J.B. \& de Kretser, D.M. (1981a) Evaluation of Leydig cell function and gonadotropin binding in unilateral and bilateral cryptorchidism: evidence for local control of Leydig cell function by the seminiferous tubule. Biol. Reprod. 24, 534-540.

Risbridger, G.P., Kerr, J.B., Peake, R., Rich, K.A. \& de Kretser, D.M. (1981b) Temporal changes in rat Leydig cell function after the induction of bilateral cryptorchidism. J. Reprod. Fert. 63, 415-423.

Seethalakshmi, L. \& Steinberger, A. (1983) Effect of cryptorchidism and orchidopexy on inhibin secretion by rat Sertoli cells. J. Androl. 4, 131-135.
Shafik, A. (1977) Anatomy and function of scrotal ligament. Urology 9, 651-655.

Taya, K., Watanabe, G. \& Sasamoto, S. (1985) Radioimmunoassay for progesterone, testosterone and estradiol-17 $\beta$ using ${ }^{125} \mathrm{I}$ iodohistamine radioligands. Jpn. J. Anim. Reprod. 31, 186-197.

Wensing, C.J.G. (1986) Testicular descent in the rat and a comparison of this process in the rat with that in the pig. Anat. Rec. 214, 154-160.

Received 4 May 1988 